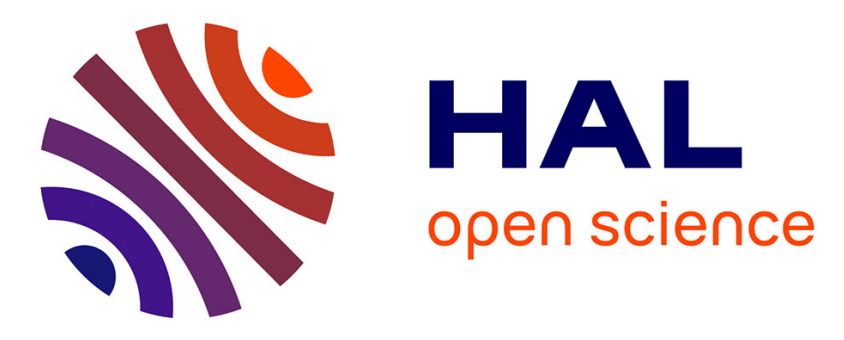

\title{
Three Dimensional Visualization of the Statically Equivalent Serial Chain from Kinect Recording
}

Alejandro González, Mitsuhiro Hayashibe, Philippe Fraisse

\section{To cite this version:}

Alejandro González, Mitsuhiro Hayashibe, Philippe Fraisse. Three Dimensional Visualization of the Statically Equivalent Serial Chain from Kinect Recording. EMBC: Engineering in Medicine and Biology Conference, Aug 2012, San Diego, United States. , 34th Annual International Conference of the IEEE Engineering in Medicine and Biology Society, pp.4843-4846, 2012, 10.1109/EMBC.2012.6347078 . lirmm-00734623

\section{HAL Id: lirmm-00734623 \\ https://hal-lirmm.ccsd.cnrs.fr/lirmm-00734623}

Submitted on 5 Dec 2012

HAL is a multi-disciplinary open access archive for the deposit and dissemination of scientific research documents, whether they are published or not. The documents may come from teaching and research institutions in France or abroad, or from public or private research centers.
L'archive ouverte pluridisciplinaire HAL, est destinée au dépôt et à la diffusion de documents scientifiques de niveau recherche, publiés ou non, émanant des établissements d'enseignement et de recherche français ou étrangers, des laboratoires publics ou privés. 


\section{Three Dimensional Visualization of the Statically Equivalent Serial Chain from Kinect Recording}

Alejandro González

\author{
Mitsuhiro Hayashibe
}

Philippe Fraisse

\begin{abstract}
We develop and present a portable tool intended for real time estimation of the center of mass (CoM) in human subjects. Using the statically equivalent serial chain (SESC) method we can account for subject specificity after identification of the model's parameters. CoM position estimates are then available from measurements of the subject's limbs orientations.

For portability, we make use of widely accessible sensors such as the Kinect and Wii balance board for identification. Use of the Kinect as a measurement device allows us to establish the SESC outside of the laboratory, without many special considerations on the environment. Only Kinect is used for CoM tracking after identification was performed.

We present here an overview of the SESC concept and the identification procedure. The aspects involved in the visualization tool are discussed and results are shown in order to verify the performance.
\end{abstract}

\section{INTRODUCTION}

Human motion stability during quiet standing and walking is concerned with the position of the subject's center of mass $(\mathrm{CoM})$ and center of pressure $(\mathrm{CoP})$. CoP can be expressed in terms of CoM's acceleration or in a simpler way, calculated from measured ground reaction forces [1]. Estimating CoM position is typically a complex issue, since it involves either body segmentation and estimation of limb weight and composition, or treatment (integration) of motion data [2], [3], [4]. Body segment data can be obtained from assumptions regarding tissue density, or by measurements performed in cadavers or living subjects. The use of literature anthropometric tables is limited to subjects similar to the sample population since CoM estimation may be inaccurate for individuals of different ages and races [2], [4]. In contrast the double integration method does not make assumptions regarding the subject but requires continuous recording of CoP position and knowledge of the CoM's initial position and velocity [3]. The difficulties of these methods could be overcome by the use of the statically equivalent serial chain (SESC) [5], [6]. This method utilizes a set of identified subject specific parameters, and a linearized model for real time estimation of CoM position from measured body segment orientations.

Simply stated, SESC creates a serial chain with its links oriented in the same direction as the subject's limbs [5], [6]. The origin of this chain could be any location of the subject's body, creating a floating base. This allows us to follow CoM throughout the subject's motion without limitations on the space.

A. González, M. Hayashibe and P. Fraisse are with INRIA DEMAR Project and LIRMM, CNRS/University of Montpellier, France. \{gonzalezde, hayashibe, fraisse\}-at-lirmm.fr
Currently, CoM estimation requires keeping track of the subject's movements. This is primarily achieved through the use of expensive motion capture equipment capable of providing limb position and orientation and/or ground reaction forces. Such environments limit the use of CoM estimation in practical and rehabilitation applications by not being widely applicable, since it requires a long set up process (such as whole body marker placement). In addition a real time display of its position may be desired as visual feedback, during balance training for example. Such a system can be used to compare CoM position with the stable bounds during both standing and walking; while giving suitable feedback to the subject. This necessity for home rehabilitation systems has motivated us to pursue a portable and versatile CoM visualization system which can also provide an accurate and real-time position estimate in unstructured environments, outside of the laboratory.

Recently, new types of widely used video game controllers such as the Kinect [7], [8], [9] and the Wii balance board [10], [11], [12] have been used for similar purposes. They have shown encouraging results after being tested, respectively, for measurement of gait characteristics and use as a rehabilitation tool for patients with balance problems.

This work aims to show the development of a tool for real time vizualition of CoM. The SESC method is chosen due to its simplicity of its calculation and subject specific nature; this method will be briefly introduced in the next section. For portability, accesibility and simplicity the Kinect and Wii board are used. The Kinect is used for marker-less tracking and to reconstruct the scene in 3D; while the Wii board is used for CoP measurement during the SESC calibration.

\section{Method}

\section{A. Statically Equivalent Serial Chain (SESC)}

It is possible to express the CoM position of any chained structure as the end effector position of an open serial virtual chain. This virtual structure is known as a statically equivalent serial chain (SESC) [5]. Using this method, finding the CoM position is equivalent to solving the direct geometric problem for the SESC.

The architecture of the SESC is dependent on the original chain. It is made up of the same number of links and they are oriented in the same direction as the corresponding link on the original chain. For any serial chained structure with $n$ links it has been found that: 


$$
\begin{aligned}
\mathbf{B} & =\left[\begin{array}{lll}
\mathbf{A}_{1} & \cdots & \mathbf{A}_{n}
\end{array}\right] \\
\mathbf{C}_{M} & =\mathbf{B R}
\end{aligned}
$$

where, in the 3D case, $\mathbf{C}_{M}$ is a 3-by-1 vector containing the position of the CoM. $\mathbf{A}_{i}$ represents a 3-by-3 matrix containing the orientation of link $i$ with respect to the reference frame. Orientations are grouped in $\mathbf{B}$, a 3-by-3n matrix. Vector column $\mathbf{R}$ describes each of the virtual chain's links.

The values of $\mathbf{R}$ may be obtained through knowledge of the chain's structure, since they contain distance terms weighted by the ratio of the mass of each link to the total mass. These values are then dependent on the mass of each link and on the location of its CoM and joint connections expressed on the link's reference frame. When the chain is composed exclusively of revolute joints, $\mathbf{R}$ contains only constant values. Interested readers may refer to [5], [6] for additional details on calculation and in-depth discussion.

If $\mathbf{R}$ is not known, it may be estimated from a set of known configurations as shown in (3). Interestingly it is possible to estimate it even when not all components of $\mathbf{C}_{M}$ can be measured. In practice, to minimize errors, a large set of measurements is obtained creating an overdetermined linear system. The system is then solved under a certain criteria, for example in the least squares method by means of the Moore-Penrose pseudoinverse.

$$
\hat{\mathbf{R}}=\mathbf{B}^{+} \mathbf{C}_{M}
$$

When one considers that an individual's arms and legs form a tree chained structure, it is possible to find a SESC which describes it [5], [6]. For identification, CoM position may be approximated by CoP readings when the subject holds a stable, non-moving pose; that is to say $\ddot{\mathbf{C}}_{M} \rightarrow \mathbf{0}$.

For the work presented here, we consider the human body to be composed of nine links connected by means of spherical joints. The SESC is described by $\hat{\mathbf{R}}$ a 27 -by1 vector. Fig. 1 shows the assumed tree-chained structure, as well as the SESC (drawn in blue) whose end effector is placed at the CoM position.

\section{B. Equipment}

1) Kinect: The Kinect sensor includes both a color and an IR camera which is capable of reconstructing depth information [7]. Although originally developed as an interaction device for console gaming, it holds great appeal as an off-theshelf cheap alternative to motion capture systems (MoCap). The Kinect realizes marker-less tracking of joint position and limb orientation. Its appeal lies on its short set-up time and the possibility of recording. Nonetheless, it is not a perfect tool. Even with no occlusions, objects close to the subject may be interpreted as part of the user, greatly reducing the tracking accuracy. For the best results to be achieved, the subject must be placed in an open area; ideally facing the sensor's cameras.

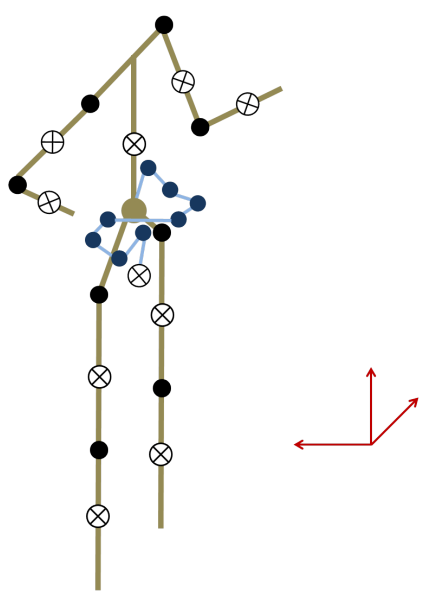

Fig. 1. Tree-chained representation of a human body and its SESC.

In the context of subject tracking for gait or motion analysis, Kinect readings are usually used to improve estimates given by other sensors, such as RGB cameras [8] or inertial sensors [9]. Measurements made with the Kinect system have been shown to be on good accordance to those provided by a MoCap VICON system [7].

We make use of the OpenNI-PrimeSense $e^{1}$ middleware and exploit its functions for creating the subject's skeleton and tracking limb orientations.

2) Wii balance board: Another off-the-shelf gaming equipment which has been the focus of increased interest from the medical and rehabilitation community is the Wii balance board. It was originally conceived as a forceplatelike controller used as an input device where the user is required to shift his or her center of pressure (CoP). To this end, the Wii-board is equipped with four pressure sensors, one at each corner. By reading force measurements on these locations, it is possible to approximate CoP position and the vertical component of the ground reaction force.

The Wii-board has been studied for use in rehabilitation settings [10], [11] and compared to laboratory-grade force plates [12] showing encouraging results during balance testing involving low speed movements.

In order to read data coming from the Wii-board, we use code provided by the open source project wiiuse $^{2}$.

\section{Visualization}

To better observe CoM motion in real time, it is useful to superpose the SESC on the video input from Kinect before displaying it for the user. The goal is to reconstruct the scene from either live or recorded depth information.

Keeping in line with the framework provided by the OpenNI-PrimeSense middleware and the wiiuse project, development is performed in $\mathrm{C}++$. 3D rendering and navigation is handled using GLUT, while OpenCV is used to perform the necessary matrix operations for CoM estimation. Fig. 2 shows an overview of the required steps during reconstruction and visualization.

\footnotetext{
${ }^{1}$ http://www.openni.org/

${ }^{2}$ https://github.com/rpavlik/wiiuse
} 


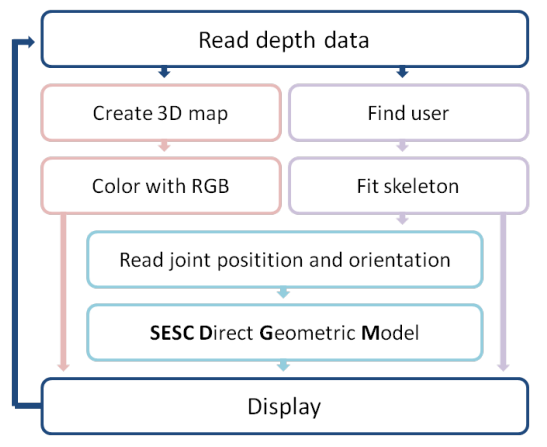

Fig. 2. Steps necessary to create 3D rendering of the scene and show CoM estimation

TABLE I

PSEUdO-CODE OF THE COM VISUALIZER.

\author{
while new frame is available do \\ if subject is detected then \\ fit skeleton \\ get joint orientation \\ compute $\mathbf{C}_{M}$ using SESC \\ end if \\ if user interaction (mouse, keyboard) then \\ change viewpoint \\ end if \\ create depth-map \\ overlay color \\ draw SESC \\ display \\ end while
}

For simplicity, it is convenient to choose the Kinect frame to serve as the world reference since all the measurements are performed with respect to it. The frame's origin is located on the camera's lens with the positive z-axis heading away from the device and the positive $y$-axis pointing upwards.

Upon reception of Kinect data, a 3D map is created based on the depth measurements. Color is added as a texture from the recorded RGB input. Whenever a subject is present, and after the calibration pose is performed, the middleware fits a skeleton, making joint positions and orientations available. This information is used in turn to find the SESC's end effector position. In order to do so, matrix $\mathbf{B}$ must be first formed from measured orientations, as such:

$$
\mathbf{B}=\left[\begin{array}{lll}
\mathbf{A}_{\text {torso }} & \mathbf{A}_{\text {hip }_{\text {left }}} & \cdots
\end{array}\right]
$$

The SESC's origin was defined on the pelvis' midpoint. CoP data used for identification was expressed as the distance between the ground projection of the SESC's origin to the measured $\mathrm{CoP}$ in the global reference frame.

Both the skeleton and SESC chains are rendered for every frame of Kinect recording. The pseudo-code of the CoM visualizer is summarized in Table I.
TABLE II

Rmse $[\mathrm{mm}]$ OF THE PROJECTED COM FROM IDENTIFIED SESC AND LITERATURE (WINTER) PARAMETERS AGAINST MEASURED COP.

\begin{tabular}{lrrrr} 
& \multicolumn{2}{c}{ Sb01 } & \multicolumn{2}{c}{ Sb02 } \\
& $x$-axis & z-axis & x-axis & z-axis \\
\cline { 2 - 5 } SESC & 23.06 & 28.67 & 20.22 & 25.47 \\
\hline Winter & 55.95 & 141.61 & 26.12 & 55.42 \\
\hline
\end{tabular}

\section{EXPERIMENTAL RESULTS}

For identification of the SESC, two able-bodied subjects were asked to stand on the Wii-board. Each subject was then asked to perform and hold a series of postures to maintain his or her CoP stable. Simultaneously, CoP and limb orientation were recorded and stored for later processing. Depth and image information were recorded from Kinect data in .oni files for reproduction at a later time, meant for CoM visualization and testing.

A total of 42 postures were used for identification. Each motion changed the CoM position in either the subject's sagital or frontal plane. A different, smaller set of poses was used for cross-validation of the results.

CoP and body segment orientation and position were used to estimate vector $\hat{\mathbf{R}}$ in the least squares sense, as described previously (3). After identification, the SESC can be used to estimate and visualize CoM position for any subject motion, providing a good orientation measurement is obtained from the Kinect. The visualization of 3D data, skeleton and SESC (Fig. 3), allows for inspection of the results making it easier to compare CoM position to that of the feet and its projection on the support polygon. The ability to navigate in the $3 \mathrm{D}$ space creates new vantage points for observing the subject's movements and the trajectory of his or her CoM.

The identified model's performance during crossvalidation tests can be seen in Table III and Fig. 4. Static $\mathrm{CoP}$ is used as reference since it is a good estimate for CoM and is readily obtained. Thanks to the characteristics of CoM calculation the SESC method should remain valid even for dynamic motions, if segment orientation can be measured. Root-mean-squared-error (rmse) of both the projected CoM from the identified SESC and that of the the antropometric parameters provided by Winter [13], against measured $\mathrm{CoP}$ were used to evaluate the identification. We find that CoM projection from the estimated SESC is closer to measured CoP position. Both the literature based and the estimated models closely follow the CoP's relative variations while absolut placement is better determined by the estimated SESC. Smaller errors were reported by Cotton et al. [6] while using the SESC method with a VICON system and laboratory force plate. Estimation errors are likely due to: 1) inconsistencies between the subject's and the model's range of motion, particularly at the trunk, 2) measurement error, or 3) an incorrect identification where not enough different postures were presented. Accuracy may be improved both by increasing the number of calibration postures and a careful data selection. 

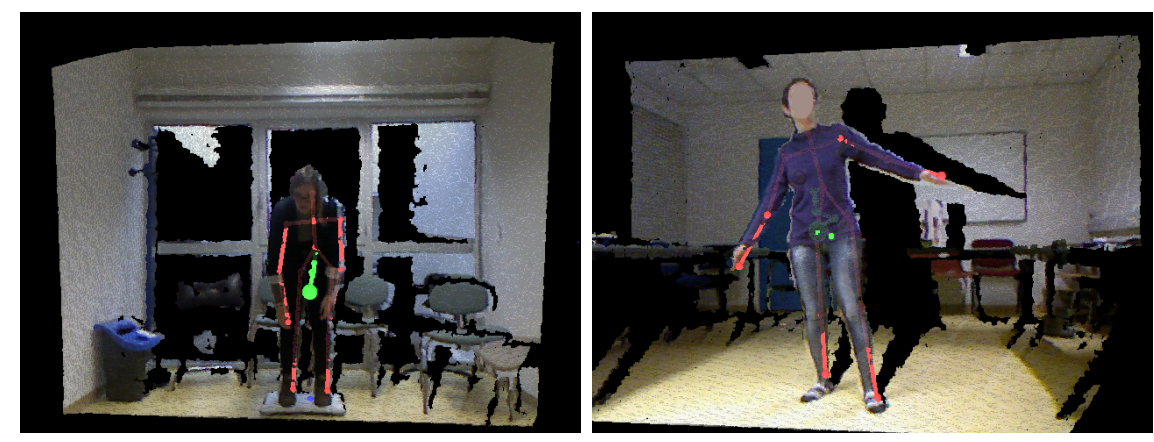

Fig. 3. 3D visualization of CoM based on SESC with Kinect user tracking.
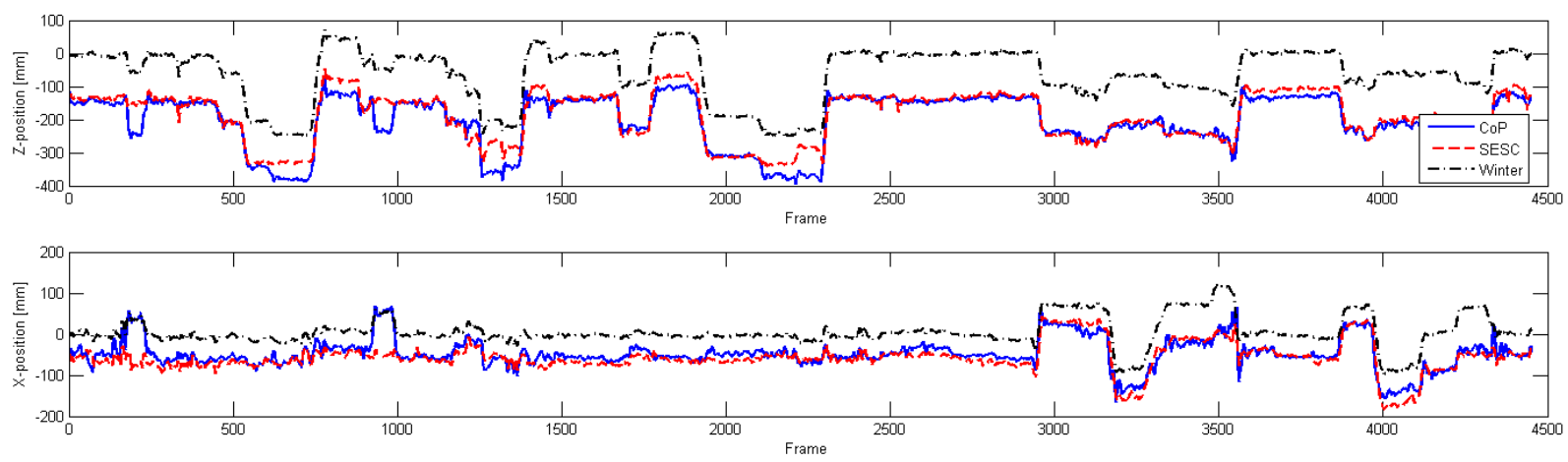

Fig. 4. Cross-validation results for Sb01. CoM projection for the identified SESC and antropometric data (from Winter) are compared to CoP.

\section{CONCLUSION}

We have developed a tool for observing and recording of a subject's CoM estimated by the SESC method. With this, we have shown that it is possible to apply the method using widely available off-the-shelf sensors such as the Kinect and the Wii balance board.

The use of Kinect results in a quicker set-up time when compared to the use of standard MoCap systems, also no other material is needed outside of the calibration phase. This allows the system to leave the structured environment of the laboratory. It would become useful in a number of situations: balance training, during rehabilitation sessions and fall prevention to name a few. Feedback can be given in real time, for a wide range of motions and without limitations regarding the subject's position.

Future work is expected to include more subjects and to add more functionalities to the visualization tool. For example; on-line parameter estimation and an evaluation of motion stability based on CoM variations aiming to prevent falls, would improve the potential of the system.

\section{REFERENCES}

[1] P. Sardain and G. Bessonnet, "Forces Acting on a Biped Robot. Center of Pressure - Zero Moment Point," IEEE Transactions on Systems, Man, and Cybernetics - Part A: Systems and Humans, vol. 34, no. 5, pp. 630-637, Sep. 2004.

[2] M. A. Jaffrey, "Estimating Centre of Mass Trajectory and SubjectSpecific Body Segment Parameters Using Optimisation Approaches," $\mathrm{PhD}$, Victoria University, 2008.
[3] H. M. Schepers, E. H. F. van Asseldonk, J. H. Buurke, and P. H Veltink, "Ambulatory estimation of center of mass displacement during walking." IEEE transactions on bio-medical engineering, vol. 56, no. 4, pp. 1189-95, Apr. 2009.

[4] P. de Leva, "Adjustments to Zatsiorsky-Seluyanov's segment inertia parameters," Journal of Biomechanics, vol. 29, no. 9, pp. 1223-1230, Sep. 1996.

[5] S. Cotton, A. Murray, and P. Fraisse, "Estimation of the Center of Mass: From Humanoid Robots to Human Beings," IEEE/ASME Transactions on Mechatronics, vol. 14, no. 6, pp. 707-712, Dec. 2009

[6] S. Cotton, M. Vanoncini, P. Fraisse, N. Ramdani, E. Demircan, A. P. Murray, and T. Keller, "Estimation of the centre of mass from motion capture and force plate recordings : a study on the elderly." Applied Bionics and Biomechanics, vol. 8, no. 2, pp. 67-84, 2011.

[7] E. E. Stone and M. Skubik, "Evaluation of an inexpensive depth camera for passive in-home fall risk assessment," Pervasive Computing Technologies for Healthcare, pp. 71-77, 2011

[8] R. Z.-L. Hu, A. Hartfiel, J. Tung, A. Fakih, J. Hoey, and P. Poupart, "3D Pose tracking of walker users' lower limb with a structured-light camera on a moving platform," in CVPR 2011 WORKSHOPS. IEEE, Jun. 2011, pp. 29-36.

[9] A. P. L. Bó, M. Hayashibe, and P. Poignet, "Joint Angle Estimation in Rehabilitation with Inertial Sensors and its Integration with Kinect," Engineering In Medicine And Biology, pp. 3479-3483, 2011.

[10] J. Deutsch, D. Robbins, J. Morrison, and P. Guarrera Bowlby, "Wiibased compared to standard of care balance and mobility rehabilitation for two individuals post-stroke," in 2009 Virtual Rehabilitation International Conference. IEEE, Jun. 2009, pp. 117-120.

[11] M. W. Kennedy, J. P. Schmiedeler, C. R. Crowell, M. Villano, A. D. Striegel, and J. Kuitse, "Enhanced feedback in balance rehabilitation using the Nintendo Wii Balance Board," in 2011 IEEE 13th International Conference on e-Health Networking, Applications and Services. IEEE, Jun. 2011, pp. 162-168.

[12] R. A. Clark, A. L. Bryant, Y. Pua, P. McCrory, K. Bennell, and M. Hunt, "Validity and reliability of the Nintendo Wii Balance Board for assessment of standing balance." Gait \& posture, vol. 31, no. 3, pp. 307-10, Mar. 2010.

[13] D. A. Winter, Biomechanics and motor control of human movement, 2005 